Journal of

Synchrotron

Radiation

ISSN 0909-0495

Received 23 December 2004

Accepted 11 March 2005

(C) 2005 International Union of Crystallography Printed in Great Britain - all rights reserved

\section{A new X-ray spectrometer with large focusing crystal analyzer}

\author{
Edmund Welter, ${ }^{\mathrm{a} *}$ Pavel Machek, ${ }^{\mathrm{b}}$ Günter Dräger, ${ }^{\mathrm{c}}$ Ulf Brüggmann ${ }^{\mathrm{a}}$ and \\ Michael Fröba ${ }^{\mathbf{b}}$ * \\ a Hamburger Synchrotronstrahlungslabor HASYLAB at DESY, Notkestrasse 85, 22607 Hamburg, \\ Germany, ${ }^{\mathbf{b}}$ Institute of Inorganic and Analytical Chemistry, Justus-Liebig University Giessen, \\ Heinrich-Buff-Ring 58, 35392 Giessen, Germany, and ${ }^{{ }^{c}}$ Fachbereich Physik der Martin-Luther- \\ Universität Halle-Wittenberg, Friedemann-Bach-Platz 6, 06108 Halle, Germany. \\ E-mail: edmund.welter@desy.de, michael.froeba@anorg.chemie.uni-giessen.de
}

\begin{abstract}
A new focusing spectrometer employing Johann geometry has been built and permanently installed at the wiggler beamline W1 at the Hamburger Synchrotron Strahlungslabor (HASYLAB) am Deutschen Elektronen Synchrotron (DESY). It is now available for user operation. The design of the spectrometer is optimized for the use of a large source spot size at the DORIS storage ring as well as for simple operation and robustness under user-mode conditions. Nevertheless, the ideal source for enlarging the number of possible applications in the future would be an undulator at one of the PETRA III beamlines. In the following the spectrometer design is described and boundary conditions such as the available energy range for future experiments are discussed. Two benchmark experiments, resonant inelastic X-ray scattering (RIXS) and X-ray absorption fluorescence spectroscopy (XAFS), on samples with complicated matrices demonstrate the performance of the new instrument.
\end{abstract}

Keywords: X-ray spectrometer; curved crystal; inelastic X-ray scattering; X-ray fluorescence.

\section{Introduction}

A focusing spectrometer of the Johann type designed for the energy range $2.1-25.0 \mathrm{keV}$ has been built and recently commissioned at HASYLAB. It will be used for high-resolution spectroscopy of inelastically scattered X-ray photons. Up to now, no spectrometer of this type has been routinely available for user experiments at HASYLAB.

A large number of investigations can be performed with the instrument, but it should be noted that the spectrometer is installed at a DORIS III wiggler beamline and therefore those applications that require extremely high brilliance (like nonresonant X-ray Raman spectroscopy) are not feasible here. In general it is considered that the spectrometer will be used in the following fields: (i) X-ray absorption fine structure (XAFS) spectroscopy in samples with complicated matrices; (ii) resonant inelastic X-ray scattering (RIXS) (Hayashi et al., 2002; Dräger et al., 2001); (iii) site-selective XAFS spectroscopy (Glatzel et al., 2002; de Groot, 2000; Izumi et al., 2000); (iv) X-ray Raman scattering (XRS) (Watanabe et al., 1996; Krisch \& Sette, 2002; Bergmann et al., 2003); (v) lifetimebroadening-removed XAFS spectroscopy (Hayashi et al., 2003).

A very good overview of most of the mentioned applications can be found in reviews by de Groot (2001) and Kotani \& Shin (2001).
In the 'classical' Johann design sample, a curved crystal and detector are arranged on the circumference of the Rowland circle. In this geometry, especially when a detector with a relatively large entrance aperture is used, the energy resolution is determined mainly by the size of the incoming beam spot on the sample (Bergmann \& Cramer, 1998). At a secondgeneration source the minimum horizontal beam size is relatively large $(\sim 4 \mathrm{~mm}$ at $\mathrm{W} 1)$ and thus negatively affects applications that demand a very high spectral resolution.

However, in our experimental set-up the horizontal beam size is not a serious limitation. Use of a two-dimensional position-sensitive detector combined with a sample position inside the Rowland circle can, on the contrary, make use of a broad beam spot because it is thus possible to map a more or less broad section of the emission spectrum onto the detector, which is, especially for the observation of weak signals, advantageous anyhow. Moreover, the loss of spectral resolution is not significant because the pixel size of the CCD chip acts like a very small detector entrance slit.

\section{Spectrometer}

During the design, emphasis was laid on easy operation in user mode and on high flexibility in the adaptation to the variety of demands of the five aforementioned applications. Use of the spectrometer in the low-energy region makes operation under 
Table 1

Technical parameters of the spectrometer.

\begin{tabular}{ll}
\hline Energy range & $2.1-25 \mathrm{keV}$ \\
Usable $\theta$ range & $20-86^{\circ}$ \\
Vacuum & $<10^{-3} \mathrm{~Pa}$ \\
Crystal bending radius & $1000 \mathrm{~mm}$ \\
Crystal diameter & $150 \mathrm{~mm}$ \\
\hline
\end{tabular}

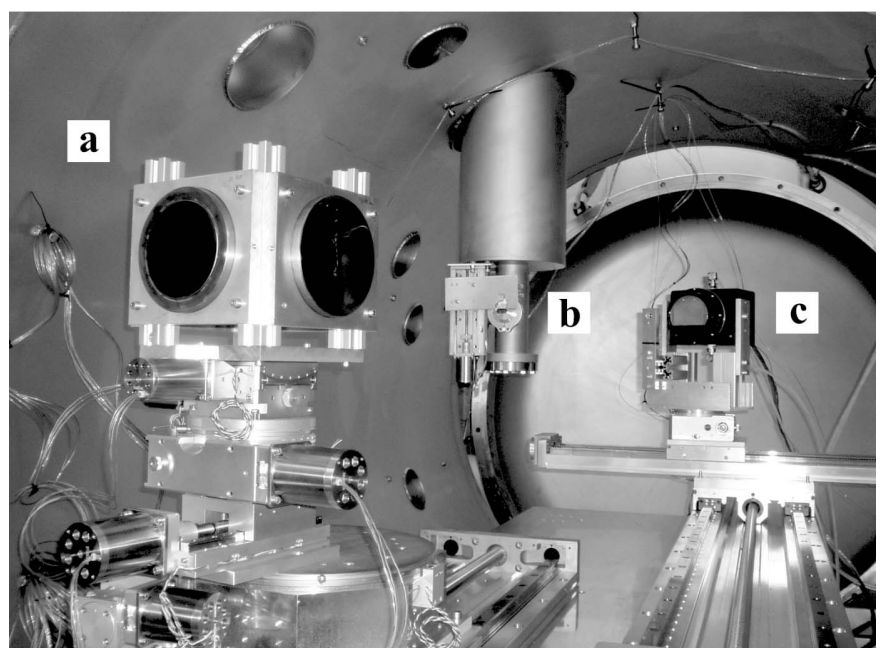

Figure 1

View into the opened vacuum tank showing the crystal holder $(a)$, sample chamber $(b)$ and CCD camera $(c)$.

vacuum mandatory. This minimizes the amount of air scattering and thus reduces the background significantly. The main parameters of the spectrometer system are summarized in Table 1.

\subsection{Mechanics}

The entire spectrometer is installed inside a large cylindrical vacuum tank (Fig. 1), which is $2.4 \mathrm{~m}$ long and has an inner diameter of $1.4 \mathrm{~m}$. The tank is mounted on three vertically and laterally adjustable posts. These movements are part of the adjustment procedure of the spectrometer and serve to position the sample and thus the entire Rowland circle with respect to the incoming beam.

The mechanical implementation of the Rowland circle is shown schematically in Fig. 2. The base of the spectrometer is a $25 \mathrm{~mm}$-thick aluminium plate, on which all the mechanical parts of the spectrometer are installed. The angle of incidence of the X-rays onto the crystal is adjusted by means of a Huber 420 goniometer equipped with a Heidenhain ROD 800 angle encoder. Large high-load linear sleds (Rexroth Star TKK 30325, TKK 20-225 and TKK 15-155) are used for the long-range linear movements of the crystal holder and the CCD camera. Their positions define the distance between the sample and the crystal and the angle $(2 \theta)$ between the sample-crystal axis and the crystal-CCD axis. Adjustment of the rotation axis of the crystal with the goniometer axis is carried out using a double-wedge goniometer head (Huber 5203.1) and a short linear stage (Huber 5101.2), which are mounted between the actual $\theta$ goniometer and the actual crystal holder.

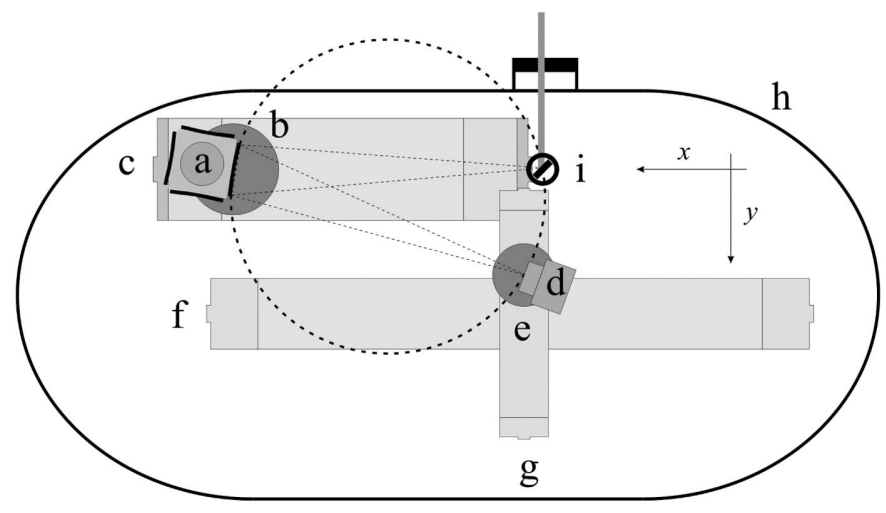

Figure 2

Schematic set-up of the spectrometer: (a) crystal holder, (b) Huber 420 goniometer, $(c)$ linear sledge for the $x$ movements of the crystal, $(d)$ CCD camera, $(e)$ Huber 409 goniometer, $(f)$ and $(g)$ linear sledges for the $x y$ movements of the CCD camera, $(h)$ vacuum tank and $(i)$ sample chamber.

The maximum feasible Bragg angle is $\sim 86^{\circ}$. This angle is constrained by the size of the sample chamber and by the width of the CCD camera enclosure. The scattered photons are collected at $90^{\circ}$ from the sample and therefore measurements with variations of the scattering angle, such as dependence of XRS (Krisch \& Sette, 2002), are excluded.

The current sample holder can hold up to six samples. They can be exchanged by remote control. The sample holder can be operated in one of two modes: either it is separated from the tank vacuum by $25 \mu \mathrm{m}$ Kapton windows or it is part of the tank vacuum. In the first case, the use of Kapton windows allows samples to be measured under ambient conditions. In the other case, two gate valves are closed in the course of a sample change so that only the relatively small volume of the sample chamber must be pumped down after the sample change. It is foreseen that cryostats and/or other specialized sample environments are to be also used.

The standard Rowland radius of the spectrometer was chosen to be $500 \mathrm{~mm}$. However, the design of the mechanical components also facilitates measurements with smaller and larger Rowland radii. In particular, a smaller Rowland radius might be interesting because it increases the solid angle covered by the curved crystal and thus the efficiency of the spectrometer. For several applications the decrease of spectral resolution, which is inevitably connected with a smaller radius, is not a problem because a resolution of some $\mathrm{eV}$ is sufficient there. Among these applications are XAFS measurements in diluted samples or in samples that produce high levels of background radiation.

The spectrometer offers a large flexibility to choose the optimum sample position on or inside the Rowland circle. This position can be changed without affecting the Bragg angle by simply moving crystal and CCD camera by means of the two large linear sledges. The measurements in a $\theta / 2 \theta$ scanning mode are not intended but are, in principle, possible.

With the sample placed on the circumference of the Rowland circle only a limited part of a fluorescence spectrum in the vicinity of energy $E$ can be detected on the detector 
position. The observable energy region $\left(E_{\max }-E_{\min }\right)$ is determined by the spot width $W_{\mathrm{S}}$ and by the Bragg angle $\theta$,

$$
E_{\max }-E_{\min }=\frac{E}{\tan \theta} \Delta \theta=\frac{E}{\tan \theta} \frac{W_{\mathrm{S}}}{R \sin \theta} .
$$

To make optimal use of the position-sensitive detector we have to move the sample into the Rowland circle. The projected spot size on the circumference of the circle is then increased and a larger energy range of the fluorescence spectra is observable (Fig. 3). The ideal distance $L$ between the sample and the circumference of the Rowland circle, in which the whole width of the CCD chip $W_{\mathrm{D}}$ is utilized for the detection of the fluorescence radiation, is found by

$$
L=R \sin \theta \frac{W_{\mathrm{D}}+W_{\mathrm{S}}}{2 r \sin \theta+W_{\mathrm{D}}} .
$$

According to (1) the observable energy range is then

$$
E_{\max }-E_{\min }=\frac{E}{\tan \theta} \frac{W_{\mathrm{D}}}{R \sin \theta} .
$$

Care must be taken so that the whole irradiated area of the sample is fully exploited as a source for reflection of photons of all energies within the energy range $E_{\max }-E_{\min }$, otherwise the resulting spectra would be distorted because of the different source size (Fig. 3) and/or inhomogeneities of the sample (Läuger, 1968).

The energy range that is observed with the sample positioned inside the Rowland circle is sufficient for most of the intended experiments. Whereas at $\operatorname{Cr} K \beta_{1,3}\left(\theta=85.855^{\circ}\right)$ the width of the CCD chip corresponds to an energy range of only about $11 \mathrm{eV}$, it corresponds to $133 \mathrm{eV}$ at $\mathrm{Cu} K \beta_{1,3}(\theta=$ $\left.62.627^{\circ}\right)$. One must keep in mind that photons with different energy are reflected from different spots on the analyzer crystal and possible local crystal defects can influence the quality of the measurements.

The energy resolution of the spherically curved crystals in Johann geometry was analyzed by Eggs \& Ulmer (1965). According to their approach, the following essential contributions to the energy resolution were identified:

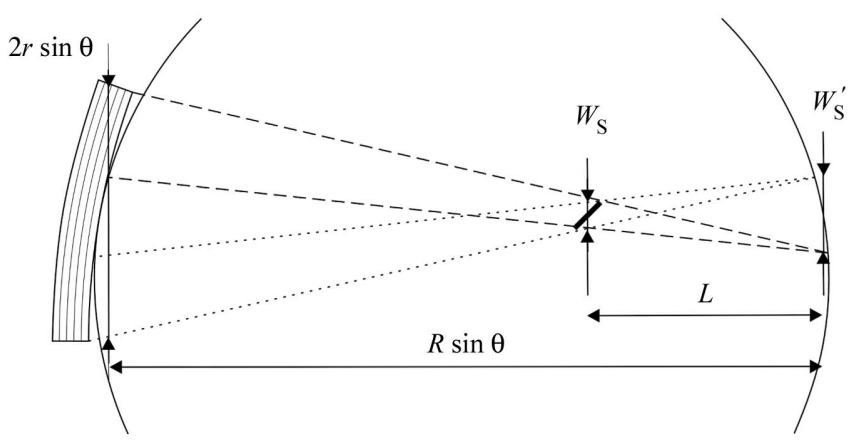

Figure 3

Detailed view of the 'inside' geometry. By moving the sample inside the Rowland circle its projected size $W_{\mathrm{S}}^{\prime}$ on the circumference of the circle is increased and a larger energy range is observable. For reliable results it is important that the whole spot size contributes to all wavelengths of the observed energy region. (a) In the Johann geometry the outer parts of the crystal with radius $r$ and bending radius $R$ are slightly deviated from the Rowland condition. This causes asymmetrical broadening $\Delta E_{a}$ towards the short-wavelength side,

$$
\Delta E_{a}=\frac{1}{2} E(r / R)^{2} \cot ^{2} \theta .
$$

For smaller Bragg angles the broadening is large $(4.67 \mathrm{eV}$ at $\mathrm{Cu} K \beta_{1,3}$ in comparison with $0.56 \mathrm{eV}$ at $\left.\mathrm{Cu} K \alpha_{1}\right)$. However, in measurements with the sample inside the Rowland circle the broadening is smaller than this value because every wavelength is reflected only from a limited part of the crystal.

(b) Reflection at spherically curved crystals results in a curved focal line instead of a point, which is caused by the vertical divergence, and furthermore is broadened owing to the vertical spot size $H_{\mathrm{S}}$. On the CCD only a part of the curved line could be seen because of the limited height $H_{\mathrm{D}}$ of the CCD chip. The resulting contribution to the energy resolution owing to the vertical divergence $\Delta E_{b}$ can be evaluated as

$$
\Delta E_{b}=E \frac{H_{\mathrm{S}}^{2}+H_{\mathrm{D}}^{2}}{8 R^{2} \sin ^{2} \theta} .
$$

(c) The CCD chip can be understood as a system of narrow slits that have a width of the pixel size $\left(W_{\mathrm{D}}=0.026 \mathrm{~mm}\right)$. The fluorescence spectrum is then measured with a resolution $\Delta E_{c}$,

$$
\Delta E_{c}=E \frac{W_{\mathrm{D}}}{R \sin \theta} \cot \theta .
$$

The energy resolution of the detector is worse at smaller Bragg angles $\left(\Delta E_{c}=0.13 \mathrm{eV}\right.$ at $\left.\mathrm{Cu} K \beta_{1,3}\right)$ than at larger ones ( $\Delta E_{c}=0.04 \mathrm{eV}$ at $\mathrm{Cu} K \alpha_{1}$ ), but still negligible in comparison with other broadening effects.

(d) Imperfections of the crystal, variations in bending and possible misalignment. These effects significantly influence the final energy resolution of the spectrometer, but cannot be evaluated directly. Their contribution was estimated by Bergmann \& Cramer (1998) to be $\Delta E_{d} \simeq 0.2 \mathrm{eV}$.

According to these equations one can estimate that the energy resolution of the spectrometer ranges between $1.0 \mathrm{eV}$ (for $\mathrm{Cu} K \alpha_{1}$ ) and $4.9 \mathrm{eV}$ (for $\mathrm{Cu} K \beta_{1,3}$ ). However, the foregoing equations give only a rough idea about the energy resolution of the spectrometer. In the following the performance of the spectrometer will be evaluated by comparisons with the results of other experiments and with theoretical calculations.

\subsection{Optics}

Spherically or cylindrically bent crystals with a maximum diameter of $150 \mathrm{~mm}$ ( 6 inch wafer) can be used as dispersive optical elements in the spectrometer. Up to four of these crystals are mounted on a revolving crystal changer. Crystal changes are thus possible without breaking the vacuum.

The spherically bent crystals were manufactured by pressing and gluing thin wafers $(0.5 \mathrm{~mm})$ cut with the appropriate crystal orientation into spherical matrices.

Currently, spherically bent Si crystals with 110, 111 and 311 orientations are available. Different crystals must be 
employed in the spectrometer because a certain crystal can cover only a limited portion of the entire energy range. Although the mechanics allows Bragg angles between $20^{\circ}$ and $85^{\circ}$, angles $\lesssim 55^{\circ}$ should be avoided because the smaller the angle becomes the larger the aberrations become. Using the higher harmonic reflexes, the currently mounted crystals already cover the major part of the desired range of operation without using Bragg angles $<55^{\circ}$ (Fig. 4).

\subsection{Detection}

Currently two CCD detectors are available. The first one is a Roper Scientific PI-LCX 1024 camera with EEV CCD36-11 deep depletion chip which is usable for energies $<15 \mathrm{keV}$. It has 1024 pixels $\times 256$ pixels, each sized $26 \mu \mathrm{m} \times 26 \mu \mathrm{m}$. The second detector is an optical CCD camera with EEV CCD3640 front-illuminated chip which has 1340 pixels $\times 1300$ pixels of size $20 \mu \mathrm{m} \times 20 \mu \mathrm{m}$. It can be used with different scintillating phosphors $(8 \mathrm{keV}$ and $17 \mathrm{keV})$ that are tapered (1:1) to the CCD chip. This camera is intended to be used at higher $\mathrm{X}$-ray photon energies.

It is foreseen that non-spatial resolving detectors such as PIN diodes or scintillation counters are also used for applications that need a larger linear working range than a CCD offers.

\subsection{Source}

The spectrometer is installed at the HASYLAB wiggler beamline W1. The critical energy of the source is $E_{\mathrm{c}}=8.1 \mathrm{keV}$. The X-ray beam is focused by the use of an Au-coated toroidal 1:0.65 focusing mirror (glancing angle $7 \mathrm{mrad}$ ). The usable energy range is $4-10.5 \mathrm{keV}$ and the energy resolution of the $\mathrm{Si}(111)$ double-crystal monochromator is about $2 \mathrm{eV}$ at $9 \mathrm{keV}$. The monochromatic flux at the sample is about $5 \times 10^{10}$ photons s${ }^{-1} \mathrm{~mm}^{-2}$. The beam size on the sample is

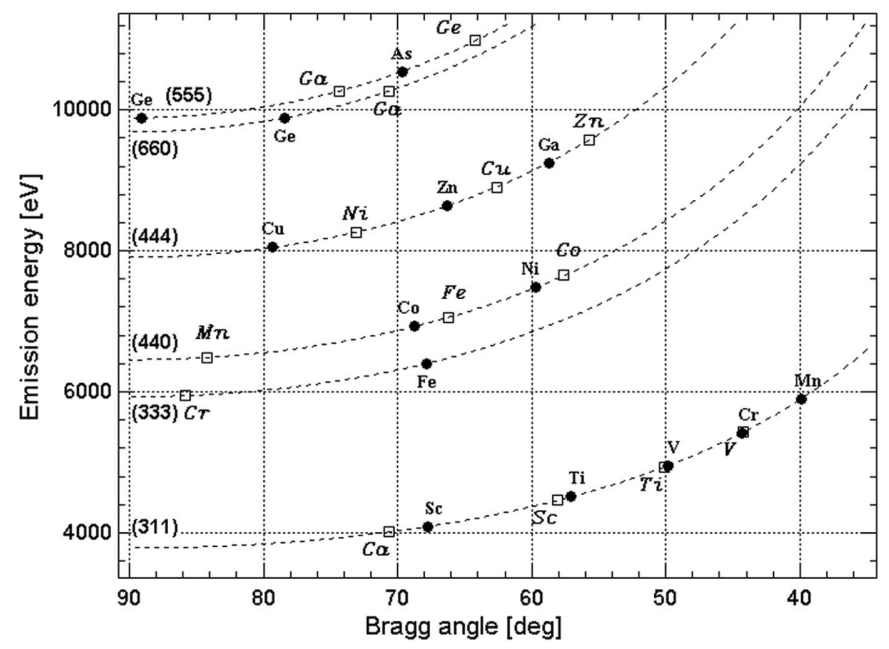

Figure 4

$K \alpha$ (black circles) and $K \beta$ (white squares) emission lines, their energies and corresponding Bragg angles. The displayed energy region corresponds to the working range at beamline W1. Measurements with Bragg angles $>55^{\circ}$ for $\mathrm{V}, \mathrm{Cr}$ and $\mathrm{Mn}$ will be possible using a crystal with 100 orientation. defined by slit systems and is typically of the order of $2 \mathrm{~mm} \times$ $4 \mathrm{~mm}$.

\subsection{Spectrometer control}

The mechanical parts of the spectrometer are controlled by the On_line software that is commonly used for the beamline operation at HASYLAB. It runs on a Linux computer that communicates with the beamline computer via a socket connection. The beamline computer runs in a slave mode and is instructed to change, for instance, the wavelength of the incoming beam by the computer that operates the spectrometer.

Data acquisition with the CCD camera is controlled by proprietary software running under Windows XP. It is nevertheless possible to communicate between the CCD controller and the software that controls the spectrometer via eight TTL input and output lines. Thus even more complex commands can be transmitted to the controller from the computer that controls the spectrometer hardware.

\section{First measurements}

The first measurements were mainly devoted to the basic adjustment of the spectrometer. After that, two of the key applications mentioned in the introduction were tested: XAFS in highly diluted or heterogeneous complex samples and RIXS spectroscopy.

\subsection{Basic alignment measurements}

The first test experiments with the complete experimental set-up of the spectrometer mounted on the base plate were performed in air. To limit air absorption and scattering to tolerable values, elements with absorption-edge energies in the upper working range of the W1 beamline were chosen. Later the spectrometer was mounted in the vacuum tank and the experiments were performed in a vacuum $\left(<2 \times 10^{-3} \mathrm{~Pa}\right)$. Together with an improved shielding of the sample, this reduced the measured background to virtually zero.

The basic adjustment was carried out optically using a point source instead of the sample and observing the reflex on the detector entrance window. Then the fine adjustment was performed using a $\mathrm{Cu}$ foil as sample and the 444 reflex of the spherically bent $\mathrm{Si}(111)$ crystal to measure the $\mathrm{Cu} K \alpha$ lines. An 'image' and the spectrum of the $\mathrm{Cu} K \alpha_{1}$ line, measured with the sample placed on the circumference of the Rowland circle and the detector in focus, are shown in Fig. 5. The maximum of the $\mathrm{Cu} K \alpha_{1}$ line was set to the tabulated value of $8047.78 \mathrm{eV}$ (the corresponding Bragg angle is $79.313^{\circ}$ ). The count rate was $2.1 \times 10^{5}$ photons s $^{-1}$ at the peak maximum. The energy of the incoming beam was $9050 \mathrm{eV}$, well above the $\mathrm{Cu} K$-edge.

The energy scale was calculated from the geometry of the experiment using the Bragg equation, assuming an infinitely small angular acceptance of the $\mathrm{Si} 444$ reflex and linear characteristic of the energy dispersion in the observed energy range. The projection of the beam size onto the sample was 
Table 2

Measured emission lines and their comparison with tabulated (tab.) values.

\begin{tabular}{|c|c|c|c|c|c|c|c|c|}
\hline & $\begin{array}{l}\text { Peak } \\
\text { energy } \\
(\mathrm{eV})\end{array}$ & Crystal & $\begin{array}{l}\text { Bragg } \\
\text { angle }\left({ }^{\circ}\right)\end{array}$ & $\begin{array}{l}\text { Width } \\
\text { utilization } \\
\text { of CCD } \\
\text { chip (\%) }\end{array}$ & $\begin{array}{l}\text { Sample } \\
\text { position in } \\
\text { Rowland } \\
\text { circle }(\mathrm{mm})\end{array}$ & $\begin{array}{l}\text { FWHM } \\
(\mathrm{eV}) \\
\text { measured }\end{array}$ & $\begin{array}{l}\text { FWHM } \\
(\mathrm{eV}) \text { tab. } \\
\text { observed }\end{array}$ & $\begin{array}{l}\text { FWHM } \\
(\mathrm{eV}) \text { tab. } \\
\text { theoretical }\end{array}$ \\
\hline $\operatorname{Cr} K \beta_{1,3}$ & $5946.70 \dagger$ & Si (333) & 85.855 & $\sim 80$ & 225 & 2.60 & $2.52 \ddagger$ & - \\
\hline $\mathrm{Cu} K \alpha_{2}$ & $8027.79 \dagger$ & Si (444) & 80.098 & $\sim 40$ & 220 & 3.43 & $2.78 \S$ & $2.17 \S$ \\
\hline $\mathrm{Cu} K \alpha_{1}$ & $8047.78 \dagger$ & Si (444) & 79.313 & $\sim 40$ & 220 & 2.36 & $2.28 \S$ & $2.11 \S$ \\
\hline Ni $K \beta_{1,3}$ & $8264.66 \dagger$ & Si (444) & 73.111 & $\sim 40$ & 220 & 5.18 & $5.44 \uparrow$ & - \\
\hline $\mathrm{Fe} K \alpha_{2}$ & $6390.84 \dagger$ & Si (333) & 68.136 & $\sim 30$ & 210 & 3.57 & $3.02 \S$ & $1.61 \S$ \\
\hline Fe $K \alpha_{1}$ & $6403.84 \dagger$ & Si (333) & 67.848 & $\sim 25$ & 210 & 2.87 & $2.60 \S$ & $1.62 \S$ \\
\hline $\mathrm{Cu} K \beta_{1,3}$ & $8905.29 \dagger$ & Si (444) & 62.627 & $\sim 30$ & 200 & 6.37 & $5.84 \ddagger$ & - \\
\hline
\end{tabular}

$\dagger$ Bearden (1967). § Sørum (1987). $\quad$ \$ Brogren (1962). Sørum \& Bremer (1982).

$7 \mathrm{~mm}$. Taking into account the resulting different Bragg angles one can calculate that the overall photon energy range that is mapped on the CCD is $\sim 6 \mathrm{eV}$ (with the photons of different energy originating from different spots on the sample). Using a Gauss fit the FWHM of the $K \alpha_{1}$ peak was determined to be $2.5 \mathrm{eV}$. This corresponds well with the tabulated values of $2.28 \mathrm{eV}$ (Sørum, 1987) and the estimated broadening. The measured peak width confirms that the slope errors of the relatively large spherically bent analyzer crystal are small enough for high-resolution measurements. Later, $\mathrm{Ni} K \beta$ (reflected at $\left.\theta=73.111^{\circ}\right), \mathrm{Zn} K \alpha\left(\theta=66.266^{\circ}\right), \mathrm{Cu} K \beta(\theta=$ $\left.62.627^{\circ}\right)$ and $\mathrm{Zn} K \beta\left(\theta=55.708^{\circ}\right)$ lines were also observed with the same crystal reflex (see Fig. 4).

Further, the $\mathrm{Cu} K \alpha_{1}$ and $K \alpha_{2}$ lines were used to test the alternative set-up with the sample inside the Rowland circle. In this geometry it is possible to see both emission lines simultaneously. In this case the spectrometer was tuned to a nominal energy between the $K \alpha_{1}$ and $K \alpha_{2}$ lines. The count rate was $2.2 \times 10^{4}$ photons s $^{-1}$ at the peak maximum of $\mathrm{Cu} K \alpha_{1}$ and $7.0 \times 10^{4}$ photons s $^{-1}$ at the peak maximum of $\mathrm{Cu} \mathrm{K \alpha _{2 }}$. The energy of the incoming beam was $9050 \mathrm{eV}$. The result is shown in Fig. 6.

Being able to see two lines of known distance made it possible to calibrate the energy axis directly and determine the width of the peaks. The measured FWHMs are $2.36 \mathrm{eV}$ for $K \alpha_{1}$ and $3.43 \mathrm{eV}$ for $K \alpha_{2}$, which are comparable with the line widths found in the literature. The intrinsic resolution of the spectrometer using the 444 reflex is obviously comparable

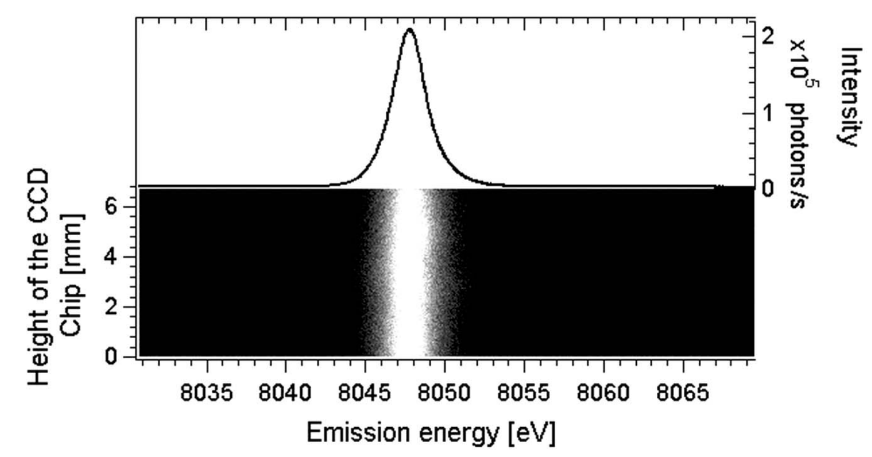

Figure 5

'Image' and spectrum of the $\mathrm{Cu} K \alpha_{1}$ line, measured with the sample on the circumference of the Rowland circle. with other experiments. The results of measurements with the sample inside the Rowland circle are summarized in Table 2.

\subsection{XAFS test experiment}

The goal of the first test experiment was to measure X-ray absorption spectra of a natural $\mathrm{CuFeS}_{2}$ (chalcopyrite) sample. The absorption spectrum of the element with higher $Z$ (here $\mathrm{Cu}$ ) is difficult to measure with an energy-dispersive detector in samples of this type because the lighter element (here Fe) produces a large background which causes problems with the overall count rate of the detector. For the detector of the focusing spectrometer the $\mathrm{Fe}$ fluorescence is irrelevant because only the photons for which the Bragg condition is fulfilled are reflected from the analyzer crystal and can reach the actual detector.

The mineral sample was ground and pressed to a pellet of diameter $13 \mathrm{~mm}$ using polyethylene as bonding agent. An XAFS scan with an accumulated measuring time per point of $3 \mathrm{~s}$ (exposure time $0.2 \mathrm{~s}, 15$ accumulations per frame) was performed. The step width was $0.5 \mathrm{eV}$ in the energy range 8970-9020 eV and $2 \mathrm{eV}$ in the energy range 9020-9050 eV.

Fig. 7 shows a three-dimensional plot of the $\mathrm{Cu} \mathrm{K} \alpha_{1}$ emission spectra measured during the excitation energy scan. The XANES (X-ray absorption near edge spectra) and first EXAFS (extended X-ray absorption fine structure) wiggles are clearly visible. The measured spectra demonstrate that the spectrometer is in principle suited for XAFS measurements.

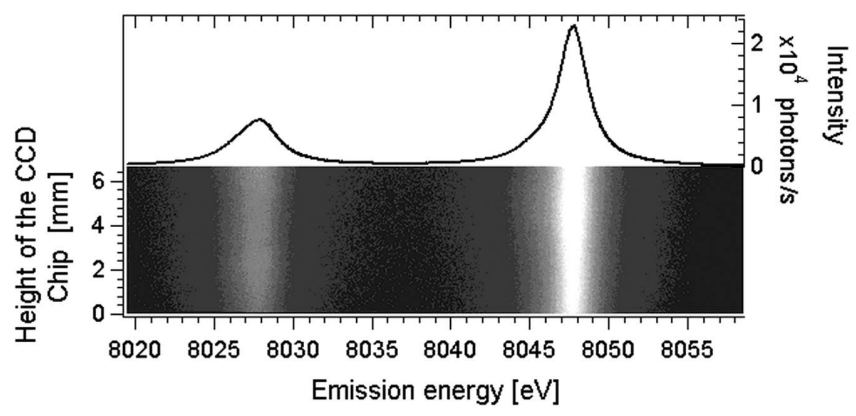

Figure 6

'Image' and spectrum of the $\mathrm{Cu} K \alpha_{1}$ and $\mathrm{Cu} K \alpha_{2}$ lines, measured with the sample $160 \mathrm{~mm}$ inside the Rowland circle. Note that almost the whole width of the CCD chip is now utilized for the measurement. 


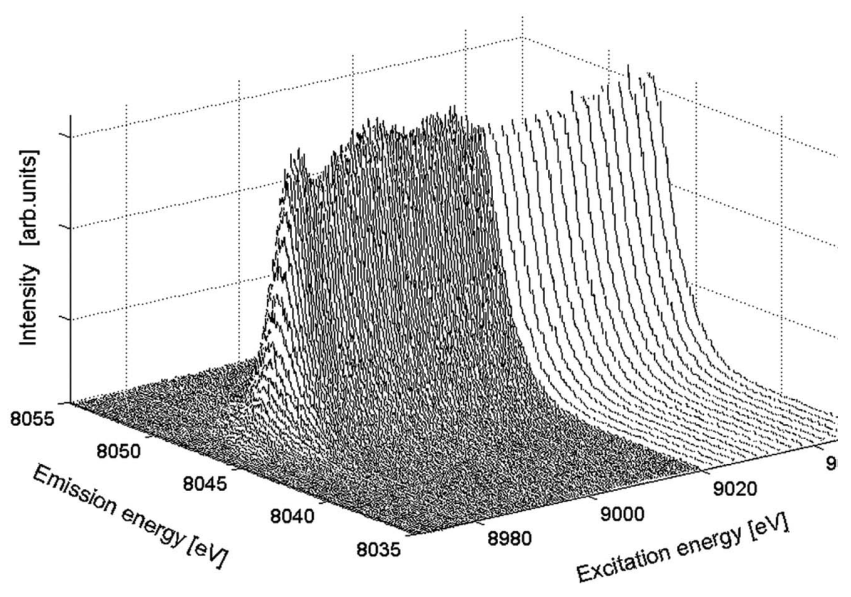

Figure 7

Three-dimensional plot of the $\mathrm{Cu} K \alpha_{1}$ emission of $\mathrm{CuFeS}_{2}$ versus excitation energy. The XANES and first EXAFS wiggles are clearly visible.

However, even in the integrated XAFS spectrum the noise was far too high with the detector used.

Two main factors were identified to contribute to the noise. The first is that the limited dynamic range and the large time needed for detector readout make a CCD an unsuited detector for XAFS measurements, because the efficiency is significantly reduced. The second is a problem of timing, as it is difficult to measure the incoming intensity and the fluorescence intensity exactly at the same time, which is necessary for the normalization of the absorption spectrum. Both problems will be tackled by the replacement of the CCD by a PIN diode with an entrance slit of suitable width. Such a detector will provide the dynamic range necessary for the registration of XAFS spectra.

\subsection{RIXS test experiment}

The second tested application was RIXS spectroscopy. $\mathrm{Cu} K \alpha_{1}$ RIXS spectra of a $\mathrm{Cu}$ (II)O sample were measured and compared with spectra that were previously measured elsewhere and published in the literature (Hayashi et al., 2003). The sample was prepared from $\mathrm{CuO}$ powder (p.a. grade, used as delivered) in the same way as the chalcopyrite sample for the XAFS experiment and it was positioned $160 \mathrm{~mm}$ inside the Rowland circle. The spectrometer was tuned to a Bragg angle of $79.313^{\circ}$ which corresponds to an emitted photon energy of $8047.78 \mathrm{eV}$ for the $\mathrm{Si} 444$ reflex. The fluorescence spectrum for each excitation energy is the average of 15 measurements, each lasting $100 \mathrm{~s}$ (exposure time $1 \mathrm{~s}, 100$ accumulations). The resulting spectra were normalized to the intensity of the primary beam. The count rates were 126 photons s $^{-1}$ at peak maximum $\mathrm{C}$ (excitation energy $8979 \mathrm{eV}$ ) and 646 photons s$^{-1}$ at $\mathrm{Cu} K \alpha_{1}$ (excitation energy $8987 \mathrm{eV}$ ).

The measured excitation energy dependence of RIXS spectra of $\mathrm{CuO}$ exhibits significant changes in the shape of the emission spectra. Whereas well above the absorption edge we can see only one maximum, which is the $\mathrm{Cu} K \alpha_{1}$ fluorescence line, in the pre-edge region we can observe three features (see Fig. 8). Feature $\mathrm{A}$ is shifted down with the decreasing exci-

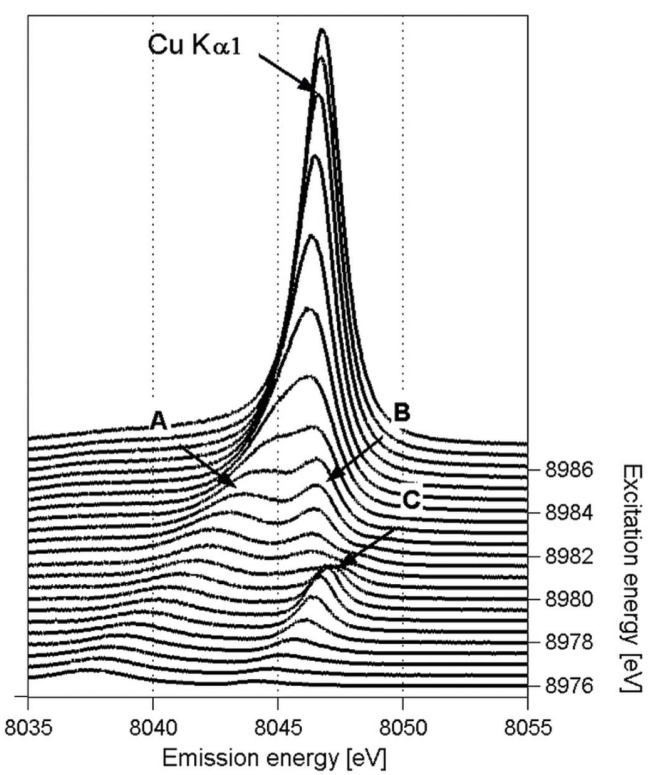

Figure 8

Excitation energy dependence of the $\mathrm{Cu} K \alpha_{1}$ fluorescence spectra of $\mathrm{CuO}$. Spectra were measured with the sample positioned $150 \mathrm{~mm}$ inside the Rowland circle.

tation energy and corresponds to the transition of a $1 s$ electron into the $4 p$ level in the first step of the RIXS process. Feature $\mathrm{C}$ also exhibits a Raman shift and corresponds to the transitions of a $1 s$ electron into unoccupied $3 d$ states. In the resonant energy region $8978-8980 \mathrm{eV}$ this feature is stronger than feature A. Feature B is prominent in the energy region 8981$8984 \mathrm{eV}$ and, as distinct from features $\mathrm{A}$ and $\mathrm{C}$, does not preserve the energy loss. This feature is unresolved in conventional XANES spectra and was recently attributed to be a $3 d$ shake-up satellite (Döring et al., 2004).

The results of our measurements are comparable with the measurements of the other authors (Hayashi et al., 2003, 2004; Döring et al., 2004) and confirmed that the performance of the spectrometer reaches the design values.

For this type of application the CCD detector proved to be very well suited, because the dynamic range is of minor importance for these relatively weak effects and the entire spectral range of interest can be measured in one shot without time-consuming $\theta / 2 \theta$ scans.

A variation of the scattering angle is not possible with the presented spectrometer. The scattering plane is identical to the storage-ring plane and thus the direction of the incident polarization and the direction of the momentum transfer cannot be kept fixed when changing the scattering angle (Döring et al., 2004).

\section{Conclusion}

A wavelength-dispersive X-ray spectrometer in Johann geometry has been built at HASYLAB. After the successful commissioning of the basic components of the spectrometer it is available for user operation at the $\mathrm{W} 1$ wiggler beamline. The feasibility of a number of experiments was proven by test 
experiments like XAFS in samples that are difficult to measure with energy-dispersive detectors and RIXS spectroscopy on a $\mathrm{CuO}$ sample. The next steps will be to test additional crystals, to build a sample holder with cryostat and to build detectors for special applications. A further improvement of the results can be expected after installation at the third-generation synchrotron radiation source PETRA III.

This research was supported by the Federal German Ministry of Research and Education (BMBF): 'Entwicklung, Aufbau und Inbetriebnahme eines hochauflösenden Fluoreszenzdetektorsystems mit einstellbarer Energieauflösung auf der Basis fokussierender Sekundär-Analysatoren für Energien von 2-25 keV' (Project No. 05KS2RG1/9).

\section{References}

Bearden, J. A. (1967). Rev. Mod. Phys. 39, 78-124.

Bergmann, U. \& Cramer, S. P. (1998). Proc. SPIE, 3448, 198-209.

Bergmann, U., Groenzin, H., Mullins, O. C., Glatzel, P., Fetzer, J. \& Cramer, S. P. (2003). Chem. Phys. Lett. 369, 184-191.

Brogren, G. (1962). Ark. Fys. 23, 219.
Döring, G., Sternemann, C., Kaprolat, A., Mattila, A., Hämäläinen, K. \& Schülke, W. (2004). Phys. Rev. B, 70, 085115.

Dräger, G., Kirchner, Th., Bocharov, S. \& Kao C.-C. (2001). J. Synchrotron Rad. 8, 398-400.

Eggs, J. \& Ulmer, K. (1965). Z. Angew. Phys. 20, 118-128.

Glatzel, P., Jacquamet, L., Bergmann, U., de Groot, F. M. F. \& Cramer, S. P. (2002). Inorg. Chem. 41, 3121-3127.

Groot, F. de (2001). Chem. Rev. 101, 1779-1808.

Groot, F. M. F. de (2000). Topics Catal. 10, 179-186.

Hayashi, H., Takeda, R., Udagawa, Y., Nakamura, T., Miyagawa, H., Shoji, H., Nanao, S. \& Kawamura, N. (2003). Phys. Rev. B, 68, 045122.

Hayashi, H., Udagawa, Y., Caliebe, W. A. \& Kao, C.-C. (2002). Phys. Rev. B, 66, 033105.

Hayashi, H., Udagawa, Y. \& Kao, C.-C. (2004). J. Electron Spectrosc. Relat. Phenom. 137/140, 277-280.

Izumi, Y., Oyanagi, H. \& Nagamori, H. (2000). Bull. Chem. Soc. Jpn, 73, 2017-2023.

Kotani, A. \& Shin, S. (2001). Rev. Mod. Phys. 73, 203-246.

Krisch, M. \& Sette, F. (2002). Surf. Rev. Lett. 9, 969-976.

Läuger, K. (1968). Thesis, Ludwig-Maximilians-Universität München, Germany.

Sørum, H. (1987). J. Phys. F, 17, 417-425.

Sørum, H. \& Bremer, J. (1982). J. Phys. F, 12, 2721-2728.

Watanabe, N., Hayashi, H., Udagawa, Y., Takeshita, K. \& Kawata, H. (1996). Appl. Phys. Lett. 69, 1370-1372. 